\title{
Le Rire tragique: Une étude de l'Ecole des femmes de Molière
}

Fayçal FALAKY

Et cosi aven che l'animo ciascuna

Sua passion sotto el contrario manto

Ricopre, con la vista hor' chiara hor bruna.'

Pétrarque

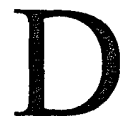

epuis l'Antiquité, les philosophes ont délibéré sur le lien apparemment paradoxal qui existe entre la souffrance et le rire. Comment est-il possible que des sensations comme la douleur et le tourment puissent susciter, voire créer, l'hilarité ? Comme l'affirme Charles Baudelaire dans De l'Essence du rire, cette contradiction « est à la fois signe d'une grandeur infinie et d'une misère infinie» (379). Les ecclésiastiques estiment que la foi est le meilleur remède pour combattre la douleur. Mais que se passe $t$-il quand les croyances religieuses perdent leur empire ? Sans foi, le mortel est désormais assujetti à voir la réalité du monde qui l'entoure, à voir l'absurdité parfois hilarante d'une vie sur laquelle la logique divine et biblique cesse d'avoir une mainmise. Le résultat de cette mutation est un étonnement souvent infantile qui plonge le sujet, à la fois, dans la consternation et dans l'émerveillement. Sans la foi, $s$ 'installe la peur d'une clarté nouvelle dénudée de toute protection divine, et contre cet éblouissement, source de peur et de savoír, mais surtout de doute, le renégat se protège par l'ivresse du rire. Comme fait 
dire Beaumarchais au personnage de Figaro voulant fuir le malheur, « je me presse de rire de tout, de peur d'être obligé d'en pleurer» (79).

Le thème du rire comme palliatif pour refuser l'adversité remonte aux débuts de la médecine occidentale. Hippocrate avait bien compris que le rire de Démocrite, loin d'être un signe de folie, fut sa recette pour ne pas tomber dans la mélancolie. Cependant, il semble que le rire ne soit pas une cure définitive contre le spleen parce que, ironiquement, il s'alimente aussi de ce qu'il est censé combattre. De la sorte, on rit pour repousser la mélancolie, mais il arrive que cette même mélancolie, germe d'un absurde "entre l'amour et le dédain $»^{2}$, peut nous faire rire. Certes, la risée mélancolique est un éclat qui dépasse la superficialité physiologique du rire. C'est une risée douloureuse parce qu'elle est essentiellement liée à la condition humaine, à la fois dans sa grandeur et dans sa misère ; c'est à dire dans sa contradiction. C'est l'humanité de l'homme qui fait rire l'homme.

Bien sûr, il faut atténuer tous ces propos. Il y a plusieurs sortes de rires, mais on se bornera dans notre étude à cerner seulement ce rire profond et ténébreux qui frôle le chagrin ; celui qui ne se proclame d'aucune certitude et qui est foncièrement plongé dans un doute à la fois pénétrant et inquiétant.

Ce rire est inlassablement contradictoire. Il peut être au service de la raison, comme dans les comédies du Castigat ridendo mores $^{3}$, ou il peut être au service de la déraison absurde. Il est à la fois du côté de l'humain et de l'animal. Dans la Beffa florentine d'Antonio di Tuccio Manetti, $I l$ Grasso legnauiolo, le peintre Brunelleschi se fait architecte d'une blague rude et violente où il consiste de persuader un gros menuisier s'appelant Manetto qu'il n'est pas lui-même mais un certain Matteo. Le burlesque de cette nouvelle de la Renaissance italienne provoque un rire primaire. Néanmoins, ce rire est accompagné d'une réflexion profonde puisque Brunelleschi conçoit la blague comme une morale de la perspective et de la relativité. Le soi n'est pas soi mais un autre ; l'identité même devient une question de point de vue.

Si on se rapporte aux comédies de mœurs du XVII ${ }^{e}$ siècle, on retrouve une approche similaire à celle de la Beffa. Même si ces comédies, bienséance oblige, manquent le côté cruel et vengeur des farces italiennes, leur ridicule est toujours accompagné par une réflexion qui peut être sociale, philosophique ou psychologique.

Quand Molière est raillé par les critiques quant à l'outrance des exclamations d'amour d'Arnolphe dans le cinquième acte de l'Ecole des femmes, le dramaturge répond par la bouche de Dorante dans la Critique del' "Ecole des femmes" : 
Et quant au transport amoureux du cinquième acte, qu'on accuse d'être trop outré ou trop comique, je voudrais bien savoir si ce n'est pas faire la satire des amants, et si les honnêtes gens même et les plus sérieux en de pareilles occasions, ne font pas des choses... ? ${ }^{4}(236-237$, II $)$

Le centre de gravité du rire ne réside plus sur un point fixe, et tout éclat devient relatif. A un premier niveau, le rire peut être dirigé contre celui qui est ridiculisé et à un deuxième (plus réfléchi) il peut retomber sur celui qui rigole.

Nous allons voir dans cette étude de l'Ecole des femmes comment cette pièce comique, représentée pour la première fois le 26 décembre 1662 , reprend un thème de la farce du Moyen Age : «A trompeur, trompeur et demi ». Mais nous allons également étudier comment ce thème dépasse la scène théâtrale, et rejaillit dans un registre différent sur le public luimême. Ceci nous aidera à éclaircir le point initial de notre étude : le lien essentiel qui existe entre la douleur et le rire.

Dans L'Ecole des femmes de Molière, Arnolphe croit qu'Agnès est idiote parce qu'elle est innocente, mais justement cette même innocence fait d'elle une fille spontanée et libre qui ne se laisse conditionner par aucune convenance sociale. La pureté des sentiments d'Agnès gagne véritablement le cœur d'Horace, qui au début ne songeait peut-être qu'à s'amuser avec elle. Elle dompte ainsi son jeune amoureux et en même temps écrase les prétentions d'un amour qui voulait l'assujettir. Par sa simplicité, Agnès triomphe de tous les pièges où l'on veut la faire tomber. Ainsi, Arnolphe, le farceur, se fait avoir. Comme dans la Farce de Maitre Pathelin, l'œuvre est construite sur un thème que traduit le proverbe : « $\mathrm{A}$ trompeur, trompeur et demi. " Elle achève également une évolution de la figure du cocu qui date de la comédie burlesque du Moyen Age. Cependant, ne nous trompons pas; la pièce de Molière est beaucoup plus qu'une simple farce. L'auteur dépasse les rebondissements, les enchaînements et les oppositions de la symétrie des tromperies qui constituent le schéma de base de la farce. Il dépasse aussi le jeu d'improvisation et les bouffonneries, « i lazzi ", qu'il avait empruntés à la commedia dell 'arte, genre dont il s'est inspiré à l'époque où il partageait la salle du Petit-Bourbon et ensuite celle du Palais-Royal avec la troupe des comédiens italiens.

Avec l'Ecole des femmes, Molière arrive à faire un mariage entre le burlesque et la réflexion. Sans abandonner le comique, il renoue ayec un théâtre classique à cinq actes basé sur ce que Baschera appelle « la tradition de la 'comédie-miroir' [et] qui a pour but de châtier les mœurs par le rire » 
(6). Ainsi, le masque de la farce prend forme et se dévoile pour montrer une nature moins superficielle, une nature qui suscite la réflexion. Soulignons que l'Ecole date d'un siècle qui est celui des Caractères de La Bruyère et des Réflexions ou Maximes de La Rochefoucauld. L'Ecole ou les Ecoles ${ }^{5}$ se situent donc dans la lignée des moralistes du XVII ${ }^{e}$ siècle. ${ }^{6}$ Les Ecoles de Molière sont censées donner une leçon.

Sur le plan idéologique, Molière dénonce une conception paternaliste de la femme. C'est une dénonciation semblable à celle que faisaient les précieuses de l'époque, et ces dernières auraient certainement applaudi la pièce de Molière si le ton trop libertin à leurs yeux avait été nuancé, et surtout si les scènes des « enfants par l'oreille » ou de «la tarte à la crème " avaient été censurées. Sur ce plan, Molière se pose aussi en moraliste moderne - ami du plaisir - qui essaie de contrecarrer les principes moraux d' " anciens » comme Bossuet, Lamoignon, voire Corneille et de précieuses qui suivent religieusement une doctrine courtoise de la vie. Même si Molière n'approuvait pas la vision chaste et chimérique qu'avaient les précieuses sur l'amour, il les rejoignait sur certains points. Dans Morales du Grand Siècle, Paul Bénichou déclare : "L'écrit qu'Arnolphe donne à Agnès, et qui, sous le titre de Maximes du mariage, doit lui enseigner ses devoirs, reproduit les interdictions traditionnelles : ni toilettes, ni fards, ni visites, ni présents, ni correspondances, ni belles assemblées, ni jeu, ni promenades.... Molière fait évidemment cause commune avec les précieuses en soutenant contre cette morale oppressive les revendications féminines » (313). La satire chez Molière attaque les vieux barbons plutôt que les jeunes lectrices du Grand Cyrus. ${ }^{7} \mathrm{~S}^{\prime}$ il y a de petites frictions avec les précieuses, il faut dire qu'il y a un désaccord total avec l'austérité des idées traditionnelles du XVII e siècle.

Cela dit, il faut souligner le lien entre l'idéologie et la dramaturgie moliéresques. Sur ce dernier plan, Molière relie la tradition comique française du Moyen Age à la tradition italienne, d'origine antique, reposant sur le conflit de générations. En fait, dans les deux Ecoles de Molière, le barbon, le vieux, se fait avoir par le blondin, le jeune. Les deux pièces sont une attaque contre la mainmise que les vieilles traditions des anciens veulent imposer à la nouvelle génération. Ce principe en fait est la force de l'intrigue comique. A la différence des pièces de Corneille, par exemple, où triomphe un sérieux tragique symbolisé par la sagesse des vieux, les œuvres de Molière donnent gagnante la légèreté nonchalante des jeunes. Ces derniers s'imposent à la fin de manière logique, voire naturelle. Les anciens, ayant déjà vécu leur vie sociale, doivent se retirer pour laisser place à une jeunesse qui veut s'exprimer librement sans l'influence pesante des pères. Cette conception de la vieillesse rappelle bien la philosophie 
de Montaigne. Ainsi, Molière utilise le rire pour ridiculiser les vieux qui s'acharnent à ne pas relâcher leur mainmise sur la société. Tel Sganarelle dans l'Ecole des maris, ils sont considérés comme des démodés, des décalés. Leurs actions sociales jadis acceptables deviennent bizarres et étrangères. C'est en effet cet aspect étranger qui provoque le rire. Dans l'Ecole des maris, Ariste reproche à son frère ce refus de s'accommoder aux usages contemporains :

Cette farouche humeur, dont la sévérité

Fuit toutes les douceurs de la société,

A tous vos procédés inspire un air bizarre,

Et, jusques à l'habit, vous rend chez vous barbare. $(566, \mathrm{I})$

Le démodé devient étranger, barbare ; et dans une France qui au milieu du XVII' siècle connaît un tournant majeur à l'égard des rapports vis-à-vis des femmes, un misogyne paternaliste devient lui aussi étranger et bizarre aux yeux de la société mondaine. Bénichou affirme : « La force des modes nouvelles dans la société française est suffisamment attestée par l'opinion générale qui faisait de la France le pays par excellence de la liberté féminine. [...] la barbarie des mœurs « turques » était universellement détestée dans les conversations de la société polie. » (315) La barbarie des mœurs turques était non seulement détestée mais également raillée, et la «barbarie » des barbons subissait la même réaction. Dans l'Ecole des femmes, Arnolphe, le riche bourgeois, tente d'accéder au statut nobiliaire en faisant une mutation onomastique -Arnolphe, étant un nom bourgeois et rappelant saint Arnoul, le patron des cocus. (Baschera 96) Cependant, son nouveau choix se révèle également fâcheux, comme le lui rappelle Chrysalde :

Ah ! malgré que j'en aie, il me vient à la bouche, Et jamais je ne songe à Monsieur de la Souche.

Qui diable vous a fait aussi vous aviser, A quarante et deux ans, de vous débaptiser, Et d'un vieux tronc pourri de votre métairie Vous faire dans le monde un nom de seigneurie ? $(92$, II)

$\mathrm{La}$ « souche » serait donc un vieux tronc pourri. Baschera nous rappelle aussi que ce nom était utilisé au XVII ${ }^{e}$ siècle pour désigner une personne lourde et stupide. Par ses démarches gauches, Arnolphe s'illustre ainsi digne de son nouveau nom, mais en plus, et surtout, il prouve par ces 
principes qu'il est un homme décalé, étranger à un milieu de plus en plus jeune que lui, mais où il veut subsister malgré tout.

On pouvait rire d'Arnolphe dans la France de la deuxième moitié du XVII ${ }^{e}$ siècle parce qu'il figurait en tant que personnage 'anormal', dans le sens propre du mot. Arnolphe, comme Sganarelle, devient un homme bizarre, un «tête de turc " si l'on veut. Cependant, dans la bizarrerie, il n'y a pas qu'un côté péjoratif. Le bizarre peut aussi être ce que Gide a appelé « l'horreur du particulier », le désir de ne pas être banal ou commun. Il y a dans le bizarre une ambition. Dans les cas de Sganarelle et d'Arnolphe, cette ambition est d'appartenir à deux mondes : celui qui convient à leur âge et un autre plus jeune, qu'ils ne veulent pas lâcher malgré le ridicule qu'ils peuvent encourir. Les deux hommes veulent faire figure de pères mais également d'amants. Ils souffrent d'un " syndrome de phénix ». Se sentant mourants en tant que pères, ils veulent ressusciter en tant qu'amants qu'ils étaient jadis. ${ }^{8} \mathrm{Ce}$ syndrome cependant se vit différemment chez les deux personnages. Sganarelle n'est pas conscient de son ambition, ou bien pire, il se croit complètement innocent tandis qu'il accuse son frère du contraire. Il dit à celui-ci : «Cela sent son vieillard qui, pour en faire accroire, / Cache ses cheveux blancs d'une perruque noire. » (567, II) Ou bien il l'appelle : « Un vieillard insensé / Qui fait le dameret dans un corps tout cassé. » (579, II) Sganarelle accuse Ariste de vouloir cacher son âge pour ne plus paraître vieux. Cependant, c'est Sganarelle lui-même qui ne veut pas abandonner des désirs juvéniles et frénétiques pour une fille qu'il est censé éduquer en tant que père. $\mathrm{Si}$ Ariste vise pendant son vieillissement à respecter la liberté de la jeune génération sans l'incommoder ni porter atteinte à ses habitudes, Sganarelle veut perturber cette liberté par sa présence permanente et incommodante. Il affirme à Valère : "Mais, savez-vous aussi, lui trouvant des appas, / Qu'autrement qu'en tuteur sa personne me touche, / Et qu'elle est destinée à l'honneur de ma couche ? " (590, II) Sganarelle ne se pose même pas la question. Il croit, malgré les objections qu'il fait à son frère, être dans son droit de redevenir amant à un âge où il lui conviendrait mieux d'agir comme tuteur. Le ridicule de Sganarelle réside dans le fait qu'il ne peut pas voir sa contradiction, ni être conscient de sa double ambition - être à la fois tuteur et galant.

Dans l'Ecole des femmes, Arnolphe est un personnage plus compliqué. Il suit la même voie ridicule que celle du frère bouffon d'Ariste, mais pourtant, il est conscient de son caprice, et il en a même honte. C'est pour cela qu'à la différence de Sganarelle, qui ne tait rien de ses exigences, Arnolphe se dissimule derrière un nouveau nom et prend même une nouvelle maison pour cacher sa bien-aimée. ${ }^{9}$ Arnolphe reconnait sa lubie, 
mais il ne peut la contrôler. Ainsi, il devient un personnage tragique, obscur, presque racinien. On dirait même qu'il a envie de faire une autopsychiatrie quand il affirme : «Ouf! Je ne puis parler, tant je suis prévenu : / Je suffoque, et voudrais me pouvoir mettre nu. » $(109, \mathrm{I})$

Le côté tragique d'Arnolphe, personnage qui est présent dans 30 des 32 scènes de la pièce, fait de l'Ecole des femmes une œuvre qui dépasse la farce et qui recèle une intrigue psychologique. Néanmoins, ce côté tragique qui fait d'Arnolphe un personnage aliéné et impuissant devant le fatum qui le maîtrise ne crée pas pour autant une tragédie. Ironiquement, ce côté ne fait qu'accentuer le comique de la pièce. Amolphe est conscient de sa double ambition et la voit même comme un combat dont le résultat ferait de lui soit un vainqueur soit un perdant. Il planifie son stratagème dès le début. Par précaution, il envoie Agnès dans un couvent pour " s'abrutir ", et une fois qu'il rencontre un ennemi sur son chemin - Horace -, il prépare tout un plan pour le décourager et ainsi le battre. En fait, Arnolphe se voit comme un maître qui veut tout contrôler. Il veut être tout : père tutélaire, pédagogue et amant, au point de dire à Agnès : " Je suis maître, je parle : allez, obéissez. " (126, II) Ce vers, pris mot pour mot de Sertorius, pièce de Corneille, exprime le décalage désespéré d'Arnolphe. Ce dernier en effet semble être un personnage cornélien, appartenant à un univers ancien et traditionnel, dans une pièce moliéresque et dans un milieu moderne qui raille le traditionalisme. C'est pour cela que malgré tous ces efforts pour apaiser ses appétits et gagner son combat, Amolphe est voué à la défaite. Il a beau vouloir tout dominer, il réalise toujours que c'est lui qui concède les défaites. Tout au long de la pièce, il se susurre des mots funestes : 'Je crève', 'Je suffoque', 'Je souffre en damné', et le fameux 'Oh'10, dernier cri d'Arnolphe avant de quitter la pièce. Ce demier 'Oh', ou 'Ouf', évoque l'ambiguìté tragi-comique du barbon de la deuxième Ecole de Molière. Dans le Panégyrique de l'Ecole des femmes, Robinet parle de ce 'Ouf'et d'Amolphe ainsi :

Le héros y montrant presque toujours un amour qui passe jusqu'à la fureur, et le porte à demander à Agnès si elle veut qu'il se tue, ce qui n'est propre que dans la tragédie, à laquelle on réserve les plaintes, les pleurs et les gémissements. Ainsi, au lieu que la comédie doit finir par quelque chose de gai, celle-ci finit par le désespoir d'un amant qui se retire avec un Ouf ! par lequel il tâche d'exhaler la douleur qui l'étouffe : dè manière qu'on ne sait si l'on doit rire ou pleurer dans une pièce où il 
semble qu'on veuille aussitôt exciter la pitié que le plaisir. (Mongrédien 209)

Robinet critique la même ambivalence tragi-comique dont Molière voulait imprégner son personnage. Robinet trouve cette ambivalence irrespectueuse des règles théâtrales, mais Molière parie sur cette nouveauté pour produire un effet galvanisant sur scène - en même temps tragique et comique. La tragédie d'Arnolphe est semblable à celle d'Alceste dans Le Misanthrope. Comme pour Alceste, c'est justement parce que l'objet de son désir lui échappe, fait preuve de résistance, qu'Arnolphe aime Agnès d'un amour violent et incompréhensible pour lui. Il désire façonner Agnès pour qu'elle l'obéisse, mais il réalise enfin que c'est lui qui est manipulé tout au long de la pièce. Il croyait posséder Agnès mais il réalise bientôt que c'est elle qui le possède. Il ne peut se passer d'elle :

Ciel ! puisque pour un choix j'ai tant philosophé, Faut-il de ses appas m'être si fort coiffé ! Elle n'a ni parents, ni support, ni richesse ; Elle trahit mes soins, mes bontés, ma tendresse : Et cependant je l'aime, après ce lâche tour. Jusqu'à ne me pouvoir passer de cet amour. Sot, n'as-tu point de honte ? Ah ! Je crève, j'enrage, Et je souffletterais mille fois mon visage. $(144$, II)

Si, jadis, il appelait Agnès une sotte, désormais Arnolphe s'interpelle comme tel. Il intègre des qualités défaitistes qu'il voulait contempler chez d'autres individus. Arnolphe ne peut plus gagner. Il est dès le début voué à l'échec parce que son ambition va contre nature, à tel point qu'il en ressent un certain besoin de repentir. Arnolphe, le quadragénaire, veut s'entremettre dans les affaires d'une génération plus jeune que la sienne. C'est dans cette tentative de s'accrocher à un corps ainsi qu'à une génération plus jeune, qu'on s'aperçoit à la fois de sa nature étrangère et qu'on réalise son aliénation. Arnolphe veut profiter des plaisirs de la jeunesse même s'il ne possède pas ses attributs ni ses principes. Arnolphe se révèle un parasite parce qu'il veut s'attacher à la jeune génération sans s'y adapter. Pire, il veut l'assujettir aux principes caducs de sa vieille époque. Subséquemment, il met son énergie au service d'une cause perdue d'avance : un bonheur conjugal garanti par l'inégalité des sexes, par la 
domination d'un sexe sur l'autre. Avant de lui lire les dix maximes du mariage, Arnolphe rappelle à Agnès :

Le mariage, Agnès, n'est pas un badinage :

A d'austères devoirs le rang de femme engage, Et vous n'y montez pas, à ce que je prétends, Pour être libertine et prendre du bon temps, Votre sexe n'est là que pour la dépendance : Du côté de la barbe est la toute-puissance. Bien qu'on soit deux moitiés de la société Ces deux moitiés pourtant n'ont point d'égalité L'une est moitié suprême et l'autre subalterne L'une en tout est soumise à l'autre qui gouverne. $(129$, II)

Ce langage archaïque fait d'Arnolphe un étranger, voire une anomalie, dans la société mondaine de la deuxième moitié du XVII" siècle. " Et comme tout corps étranger peut susciter le rire collectif, Arnolphe dans l'Ecole des femmes provoque, tel un " turc et ses lois » le rire de la société polie française.

Dans Psychocritique du genre comique, Charles Mauron affirme que « c'est toujours par rapport à l'infantilisme dépassé que le triomphe se mesure » (20). L'homme qui reste enfantin est une personne incapable de s'adapter aux normes de la société adulte. Il commet des fautes infantiles - des fautes qui transgressent la norme convenable, et qui peuvent provoquer le rire. La société raille les idées immatures parce qu'elles sont étrangères au conformisme social - elles sont étrangères aux idées communes. Mauron continue :

L'estimation de la différence implique une identification rapidement consentie puis niée : « Je pourrais être cet autre, mais je ne le suis pas ». Bien souvent, en effet, le railleur a connu l'état du raillé et en refoule le souvenir. Ainsi s'établit une continuité entre le rire triomphal du bébé (rire tout personnel, même s'il exige la connivence d'autrui) et la moquerie proprement dite, impliquant un âge mental plus avancé. (21-22)

On peut dire que la raison pour laquelle on se moque des actions d'Arnolphe est qu'elles sont infantiles - sottes. Arnolphe, le barbon, serait-il un enfant ? D'un point de vue philosophique, on peut dire que oui. Montaigne n'avait-il pas laissé entendre que la vieillesse était une 
deuxième enfance ? Cependant, le public ne peut pas réagir aux étourderies d'Arnolphe de la même manière qu'il réagirait aux puérilités d'un jeune débutant niais. Pour un public moyen, ni vieux ni jeune, il peut bien se dire dans le cas du jeune débutant : « J'aurais pu être cet autre, mais je ne le suis pas ", et ensuite pleurer d'un rire triomphal. Dans le cas d'Arnolphe, le rapport se complique. Comme dans le cas du jeune débutant, on peut bien accuser le barbon d'infantilisme. Cependant il n'est pas sûr qu'on le fasse avec le même triomphalisme éprouvé. L'âge d'Arnolphe ne nous permet pas d'avoir la même assurance. On ne peut pas se comparer à lui de la même façon qu'on le fait avec le jeune, soit parce qu'on n'a pas encore atteint l'âge du barbon, soit parce qu'on est déjà aussi âgé que lui.

Les quadragénaires parmi le public de Molière pourraient, même s'ils les trouvent ridicules, s'identifier aux tourments d'Arnolphe. Rappelonsnous la réplique de Molière aux critiques qu'on lui a faites à propos du cinquième acte :

Et quant au transport amoureux du cinquième acte, qu'on accuse d'être trop outré ou trop comique, je voudrais bien savoir si ce n'est pas faire la satire des amants, et si les honnêtes gens même et les plus sérieux en de pareilles occasions, ne font pas des choses...? $(236-237$, II)

Pour les jeunes cette identification n'est pas possible. Au contraire, ils savoureraient sans doute la défaite du barbon. Cependant, puisque leur rire n'est pas un signe de triomphalisme par rapport à une enfance dépassée mais plutôt un éclat contre une étape de la vie qui est toujours à venir, ce rire ne peut être purement comique. Mauron dit que le triomphe se mesure par rapport à l'infantilisme dépassé. Arnolphe ne peut plus avoir l'espérance de ce dépassement. Il ne peut pas dépasser sa condition, puisqu'il n'est pas dans une phase ascendante, progressive de sa vie. Arnolphe vit plutôt dans un compte à rebours. Il est de l'autre côté du spectre. Plus le temps passera, plus il sombrera dans la sénilité/puérilité. Donc, le rire que provoque Arnolphe est un rire doublement cruel et digne d'une comédie tragique. D'abord, on raille un personnage qui n'a plus d'espoir de dépasser son état de raillé. Au contraire, il y sombrera de plus en plus. Ensuite, en raillant Arnolphe, on ne se moque pas de ce qu'on aurait pu être, mais plutôt de ce qu'on pourrait devenir. Notre première enfance dépassée, on doit s'attendre aux surprises que notre deuxième enfance va nous préparer.

Ainsi, la symbolique d'Arnolphe dépasse son propre personnage. Il devient un objet de raillerie capable de nous hanter. Arnolphe peut 
représenter notre propre obsession de ne pas vouloir devenir comme lui - ou pire encore, il peut incarner la peur terrifiante d'être destiné à suivre son chemin. Molière, l'auteur, n'est-il pas en fait le premier à suivre la voie d'Arnolphe ? Molière qui jouait le rôle du barbon avait, comme ce dernier, à l'âge de quarante ans, demandé la main d'une fille vingt-trois ans sa cadette. Le mariage avec Armande Béjart, fille de Madeleine, mariage pas très heureux, a eu lieu en 1662, la même année où fut jouée pour la première fois l'Ecole des femmes. Ce mariage avec la fille de sa maîtresse lui vaut d'être accusé de relations incestueuses avec une femme qui pourrait bien être sa fille. Le rapport avec la vie d'Arnolphe, qui lui aussi veut épouser « sa propre fille », est étonnant et ne peut être une simple coïncidence. Molière serait-il lui aussi un barbon ? Il le prouverait bien si on ne faisait que citer cette phrase de l'Impromptu de Versailles, pièce où Molière joue son propre rôle. Il dit à Armande, Mademoiselle Molière : « Taisezvous, ma femme, vous êtes une bête » $(252$, II). Molière devient-il le barbon dont il se moquait et dont il se moque toujours ? Retombe-t-il dans l'infantilisme et, comme Arnolphe, en souffre-il ? Les témoignages sur ses tentatives infructueuses pour contrôler une fille issue d'une nouvelle génération le prouvent. Comme Arnolphe, il sera cocufié et raillé. ${ }^{12}$

En conséquence, on ne peut affirmer, comme le fait René Jasinski, que Molière ait des porte-paroles dans ces pièces. Molière n'utilise pas Chrysalde, Ariste ou Philinte comme vecteurs de sa philosophie. Molière s'exprime d'une manière ou d'une autre à travers tous ces personnages. Ainsi, diviser les personnages entre avocats de la morale et naturalistes (ceux qui prennent parti pour les plaisirs de la vie) ne peut être une tâche facile ni apparente. D'une certaine manière, tous les personnages de Molière, même les barbons, sont naturels. Dans Morales du Grand Siècle, Bénichou affirme : "Molière, dans la mesure où il pense que l'instinct naturel gouverne la vie, ne pense pas autrement que les barbons qu'il ridiculise. Arnolphe et ses pareils, loin de nier la force des tentations, en sont littéralement obsédés : " la chair est faible » est leur axiome principal » (313).

Donc, que cela soit par le biais d'Agnès ou d'Arnolphe, Molière n'arrête pas de se manifester-parfois il le fait de manière légère et comique, d'autres fois de façon plus douloureuse, plus tragique.

Au début de l'Ecole des femmes, le public se moque davantage d'une jeune sotte-Agnès. Elle fait rire par son ingénuité. On pourra dire qu'elle n'a pas encore dépassé son infantilisme. Alors, comme on fait souvent avec les enfants, Arnolphe la met en scène pour se moquer de ses maladresses. En raillant l'innocence d'Agnès, Arnolphe se veut et se voit maître. Cependant, Agnès arrive à dépasser son infantilisme. Grâce à 
l'amour qu'elle éprouve pour Horace, Agnès se révèle. Elle devient une personne indépendante qui a confiance en son propre jugement : « Je n'entends point de mal dans tout ce que j'ai fait (172, II). Elle arrive même à mettre en scène Arnolphe en le laissant croire qu'elle est encore naïve, ignorante, et illettrée. La lettre d'amour qu'elle écrit à Horace est - malgré l'innocence - une des plus raffinées qu'on peut imaginer. Ainsi, elle assujettit le maître et désormais, c'est Arnolphe qui tombe dans l'infantilisme.

Cette chute est comique puisque le railleur est raillé, mais elle est aussi tragique. C'est une chute - à résonance biblique - sans espoir ni récupération puisqu'il n'y a pas de retour possible (on ne peut pas remonter le temps). C'est une chute aussi cruelle que les dernières paroles de la victime avant qu'il sorte pour ne plus réapparaître : l'Ouf d'Arnolphe commenté par Robinet. ${ }^{13}$

Il n'y a aucun doute que l'Ecole des femmes est, par son style, une comédie. Cependant, on ne peut ignorer tous les traits profondément tragiques qui font de cette pièce la première des grandes comédies de Molière, c'est-à-dire la première qui dépasse le domaine de la farce et du burlesque simple.

Le rire dans l'Ecole des femmes est donc une source d'amusement primaire mais aussi d'une réflexion sur la condition humaine. Brunelleschi l'avait compris ; c'est une question de perspective. Les spectateurs peuvent bien railler le ridicule d'Arnolphe, mais, une fois intériorisée, cette raillerie ne peut se faire sans une arrière-pensée amère. Alors, face à la complexité d'Arnolphe, personnage burlesque mais troublant, le spectateur découvrira une sensation (comme disait Pétrarque) « tantôt joyeuse, tantôt sombre. »

New York University

\section{Notes}

1. « Et c'est ainsi que l'âme ouvre ses passions sous des apparences contraires, le visage tantôt joyeux, tantôt sombre. " Montaigne cite ces vers extraits du Canzionere de Petrarque dans l'essai intitulé « Comme nous pleurons et rions d'une même chose. "

2. Dans le poème qui s'intitule Clotilde, Apollinaire écrit :

L'anémone et l'ancolie

Ont poussé dans le jardin

Où dort la mélancolie

Entre l'amour et le dédain 
3. «La comédie châtie les mœurs en riant ", devise latine forgée par Jean-Baptiste Santeuil et adoptée par les Comédien italiens ainsi que par Molière.

4. Des choses... pareilles? C'est ce que Dorante voualait sans doute dire mais elle est interrompue par le Marquis qui lui demande de se taire.

5. Il ne faut pas oublier l'Ecole des Maris, pièce en 3 actes écrite en 1661 , une année avant l'Ecole des Femmes. Molière n'est pas le premier à employer le mot. Il l'a emprunté à Dorimond, auteur de l'Ecole des cocus ou la Précaution inutile.

6. Comme le prétend Baudelaire, un anti-moraliste peut bien être un moraliste lui aussi.

7. Roman précieux de Madeleine de Scudéry.

8. A quarante-deux ans, Arnolphe est certes, selon les mœurs du XVIIe siècle, un homme plutôt vieux. Son désir d'épouser Agnès n'a pourtant rien de scandaleux. Ses mariages disparates étaient assez courants à l'époque. Il faut aussi se rappeler que Ariste qui avait 60 ans et Sganarelle, les deux personnages de L'Ecole des Maris, étaient plus âgés que lui. Cela dit, ce n'est pas l'âge mais le comportement d'Arnolphe qui fait de lui un vieillard, un barbon.

9. Bien sûr, on peut dire que ce désir de cacher Agnès est dû à sa peur de devenir cocu, mais cela serait un manque de perspicacité qui nous limiterait à un niveau de lecture élémentaire.

10. 'Ouf'selon certains textes.

11. Ce langage n'est archaïque que dans la société mondaine. Il demeure cependant le langage officiel de l'Eglise et de la loi. Cet écart explique une partie de la querelle qui va suivre la pièce et éclaire en parti le point de vue de notre étude : l'aliénation (due à l'anachronisme) peut susciter le rire et la pitié.

12. Les similitudes entre la vie de l'auteur et celle de son personnage sont tellement troublantes, il est difficile d'imaginer que Molière n'est pas en train se peindre de manière tout à fait consciente. Il faut aussi rappeler que Molière épouse Armande Béjart, la fille de Madeleine, la même année où il écrit l'École des femmes.

13. Soulignons qu'il y a une certaine lueur d'espoir à la fin du Misanthrope. Alceste dit :

Trahi de toutes parts, accablé d'injustices,

Je vais sortir d'un gouffre où triomphent les vices ;

Et chercher sur la terre un endroit écarté,

Où d'être homme d'honneur, on ait la liberté. 


\section{Ouvrages cités}

Apollinaire, Guillaume. Alcools. Paris: Gallimard, 1920.

Baschera, Marco. Théâtralité dans l'œuvre de Molière. Biblio 17108. Tübingen: G. Narr Verlag, 1998.

Baudelaire, Charles. Euvres Complètes, ed.. J. Crépet. Paris: Louis Conrad, 1923.

Beaumarchais, Pierre-Augustin Caron de, Euvres complètes. Paris: Firmin Didot frères, 1865.

Benichou, Paul. Morales du Grand Siècle. Paris: Gallimard, 1948.

Jasinski, René. Molière. Paris: Hatier, 1969.

Mauron, Charles. Psychocritique du Genre Comique. Paris: Librairie José Corti, 1964.

Molière. Théâtre Complet, Ed. Pierre Malandain. 2 vols. Paris: Imprimerie nationale, 1997.

Mongredien, Georges. La Querelle de l'École des femmes. Paris: Librairie Marcel Didier, 1971.

Robinet, Charles. Panégyrique de l'Ecole des femmes.1664. Ed. G. Mongrédien, La Querelle de l'Ecole des femmes. Paris: Librairie Marcel Didier, 1971.

\section{Ouvrages consultés}

Adam, Antoine. Histoire de la littérature française au XVIIe siècle. Paris: A. Michel, 1997.

Bray, René. Molière, Homme de théâtre. Paris: Mercure de France, 1954. Defaux, Gérard. "Sagesse et folie d'Erasme à Molière." Modern Language Notes 91 (1976): 655-671.

Eustis, Alvin. Molière as Ironic Contemplator. Paris: Mouton, 1973.

Forestier, Georges. Molière en toutes lettres. Paris: Bordas, 1990.

Gossman, Lionel. Men and Masks : A Study of Molière. Baltimore: Johns Hopkins Press, 1963.

Gutwirth, Marcel. “Arnolphe et Horace." L'Esprit créateur 6 (1966): 188196.

Kern, Edith. " $L$ 'Ecole des femmes and the spirit of farce." $L$ 'Esprit créateur 13.3(1973):220-228.

Magne, Bernard. " $L$ 'Ecole des femmes, ou la conquête de la parole." Revue des Sciences humaines 145 (1972): 125-140.

Manetti, Antonio. La Novella del Grasso Legnaiuolo. Milano: Garzanti, 1998. 
Picard, Raymond, "Molière comique ou tragique? Le cas d'Arnolphe." Revue d'Histoire Littéraire de la France 6 (1972): 769-785. 
